\title{
Identification of Candida species and Antifungal Sensitivity Testing by Disc Diffusion Method
}

\author{
Mangala S. Harbade, Jyoti A. Iravane*, Shravani Kante and Nishigandha Pedwal \\ Department of Microbiology in Mycology section of Government Medical College \\ Aurangabad, India \\ *Corresponding author
}

\section{A B S T R A C T}

\begin{tabular}{|c|}
\hline Keywords \\
\hline $\begin{array}{l}\text { Candida species, } \\
\text { Antifungal } \\
\text { sensitivity }\end{array}$ \\
\hline Article Info \\
\hline $\begin{array}{l}\text { Accepted: } \\
17 \text { October } 2020 \\
\text { Available Online: } \\
10 \text { November } 2020\end{array}$ \\
\hline
\end{tabular}

Candida is an opportunistic fungi responsible for causing Candidiasis when the patients immunity is low. Emergence of Nonalbicans Candida along with Candida albicans is also increasing. Increased use of antifungal drugs, use of antibiotics, long term use of catheters has contributes to resistance to antifungal drugs. Isolation of Candida species by conventional and Hichrome agar was done. Antifungal sensitivity testing was done by disc diffusion method. Aim of the study is to identify the Candida species from various samples by conventional method and on Hichrome agar; To do the antifungal sensitivity of Candidial species by disc diffusion method. Total samples received in mycology section in microbiology laboratory were 138. Out of these 102 shows Candida species. All these Candida species were grown on Conventional methods and Hichrome agar. Among the 102 Candida isolates, Candida albicans were $47(46 \%)$ and Non albicans Candida were 55 (53.9\%). In non albicans Candida, Candida tropicalis 33 (32.3\%) Candida glabrata 13(12.7\%), Candida krusei 5(4.9\%), Candida parapsilosis 3(2.9 \%) and Candida dublinesis $1(0.9 \%)$ were found. All candida species grown on Conventional method equally grow on Hichrome agar Candida albicans was commonest isolates in Urine and sputum samples. Whereas Candida tropicalis was commonest isolate in Blood cultures, Pus, Tracheal aspirate and CSF. Anifungal sensitivity of Candida species was done by Disc diffusion method against Amphoterecin B, Flucanozole and Itracanozole. Amphoterecin showed 100\% sensitivity to Candida species. Resistant was seen in Candida albicans and Candida tropicalis to antifungal drugs. Other species does not show resistance. Candida albicans showed resistance to Itracanozole 22(47\%) and Flucanozole 17(36\%). Candida tropicalis showed resistance to Itracanozole 6(18\%) and Flucanozole 5(15.1\%). Candida albicans was the commonest isolate followed by Candida tropicalis. Nonalbicans Candida was reported at higher rate than Candida albicans. Hichrome agar is rapid method for identification of Candida species .Resistance to antifungal agents is seen in Candida species. Resistance to antifungal drugs for Itracanozole and Flucanozole by Candida albicans and Candida tropicalis is noted. The other Candidial species does not show resistance. Identification of correct Candida species and antibiotic sensitivity testing is necessary for correct treatment. Candida albicans and Nonalbicans Candida are also responsible for causing hospitalized infection. Hichrome agar gives early identification of Candida species. Resistance to azole group of antifungal is noted. 


\section{Introduction}

Candida is opportunistic fungi, normally present as a part of human anatomical flora, however they are capable of causing infection in presence of opportunistic as low immunity (Roopa et al., 2015). Candidiasis is the most common fungal disease in humans caused by Candida, yeast like fungi that produce pseudohyphae. Production of Pseudohyphae indicates active and invasive infection. It affect skin, mucosa and various internal organs of body. Different species of Candida are known such as Candida albicans, Candida tropicalis, Candida glabrata, Candida krusei, Candida parapsilosis, Candida dubliniensis, Candida kefyr, Candida guilliermondii and Candida. viswanathii. (Shivaprakash et al., 2007) Candida albicans is the commonest species causing Candidiasis uptill now, but the trends have changed to non-albicans Candida (NAC) is increasing (Sardi et al., 2013) and (Lewis et al., 2009).

Immunity is lowered by various factors like indiscriminate use of antibiotics, AIDS and other immunosuppressive conditions have led to significant rise in Candida and other fungal infections (Ishan et al., 2019).

Identification of Candida species can be done by conventional methods and by using HiCHROME agar. Isolation of Candida species is very important from an infection in order to administer appropriate therapy to reduce mortality, to control outbreaks and to carry out epidemiological investigation (Sanglard et al., 2016).

There is emerging resistance of antifungal drugs to candida species. Indiscriminate use of azole group of drugs leads to resistance. Therefore Antifungal sensitivity testing is essential for administrating accurate antifungal drugs (Morrell et al., 2005).

\section{Materials and Methods}

The study was conducted in Department of Microbiology in Mycology section of Government Medical College Aurangabad from July 2019 to July 2020.Samples of all age group with Clinically suspected Candidal infection were collected in OPD and ward and were send to Mycology section for processing. Also the samples processed in Bacteriology laboratory showing growth of Candida were also included in our study. Various samples which were processed were Urine, Blood, Sputum and Pus, Nail scraping and tracheal aspirate.

From samples direct microscopy using 10\% $\mathrm{KOH}$ and Gram stain shows gram positive oval yeast cells. Samples was Inoculated on Sabourauds dextrose agar (SDA). Next day on SDA colonies of Candida species were small, $1-2 \mathrm{~mm}$ creamy white smooth, pasty were seen. Again from colonies gram stain was done (gram positive oval budding yeast with or without pseudohyphae). Candidial species were identified by germ tube test and on corn meal agar. If Germ tube is Positive than presumptively identify as Candida albicans and Candida dubliniensis. If germ tube is negative than other Candidial species are suspected. Identified Candida species were verified by growing them on Hichrome agar by characteristic colony color. Chromogenic media contain chromogenic substrates which react with enzymes secreted by target microorganisms to yield colonies of varying colors. Hichrome medium can be used as selective medium for growing Candida species in less than 48 hours.

Colonies from SDA were inoculated on Hichrome agar and kept for incubation at 37degree centrigrade. The species were identified by characteristic colony color. Identification of Candida species by seeing the color of colonies as follows. 
Candida.albicans - Light green colored smooth colonies.

Candida.tropicalis - Blue to metallic blue colored raised colonies.

Candida.glabrata - cream to white smooth colonies.

Candida. krusei - Purple color.

Candida. dubliniensis- dark green. (HiMedia Laboratories Pvt. Ltd. HiCrome Candida Differential Agar M1297A; 2011.)

Antifungal susceptibility testing was done by disc diffusion method as recommended by CLSI M-44 A2 guidelines.9. (CLSI, 2009).

Muller Hinton agar with $2 \%$ glucose and $0.5 \mu \mathrm{g} / \mathrm{ml}$ methylene blue was used.

Following antifungal discs were used

1. Fluconazole $10 \mu \mathrm{g} /$ discs, (Himedia lab.Pvt Ltd)

2. Itraconazole $10 \mu \mathrm{g} /$ discs (Himedia lab.Pvt Ltd)

3. Amphotericin B $20 \mu \mathrm{g} /$ discs (Himedia lab.Pvt Ltd)

The standard strains used were Candida albicans ATCC 90028, Candida tropicalis ATCC 750, Candida parapsilosis ATCC 22019, Candida krusei ATCC 6258. Candida glabrata ATCC.

Procedure- For antifungal sensitivity testing Inoculum suspension was prepared by taking Five colony from Sabourauds dextrose agar and suspended in $5 \mathrm{ml}$ of sterile normal saline. The resulting suspension was vortexed for 15 seconds. The cell density was adjusted with spectrophotometer by adding sufficient sterile saline to increase the transmittance to that produced by a 0.5 Mcfarland standard at 530 NM wavelengths.

\section{Inoculation of test plates}

The plates were inoculated as per standard CLSI guidelines and incubated at 35 degree C. Zone diameters in millimeter for the zone of complete inhibition were determined after 24 hours of incubation. When insufficient growth was encountered at the hour reading the plates were re-evaluated after a further 24 hours.

\section{Results and Discussion}

Total 138 samples were received. Out of 138 total samples Candida isolates were seen in $102(73 \%)$ and $36(26 \%)$ sample were sterile.

Table 1 shows the age groups of patients showing Candida isolates

As shown in Table 1 maximum Candida isolates were found in above 50 years of age group patients that is $39(38.2 \%)$ and less in 10 to 30 years of age that is $10(9.8 \%)$.

In babies and age groups of 40 to 50 years Candida isolates were $12.7 \%$.

Among the nonalbicans Candida, Candida tropicalis was $33(32.3 \%)$ in maximum numbers followed by Candida glabrata 13(12.7\%), Candida krusei 5(4.9\%), Candida parapsilosis 3(2.9 \%) and Candida dublinesis 1 (0.9\%).

In table 2, out of 102 showing Candida isolates urine samples were $56(54.9 \%)$, blood were 19 (18.6\%), sputum were 11 $(10.7 \%)$, Pus $8(7.8 \%)$ and $4(3.9 \%)$ were from CSF and Tracheal aspirate. Candida albicans was maximally isolated species in urine and sputum samples. In urine sample Candida albicans was isolated in 29 (52\%) followed by Candida tropicalis, Candida 
glabrata, Candida krusei, Candida parapsilosis and Candida dublinensis. Most of the patients were catheterized. Among the total 102 samples $48(47 \%)$ patients were catheterized and 58(56.8\%) patients were not having catheter. In11 total sputum samples Candida albicans 7 (64\%) were in maximum.

In Blood cultures, Pus, Tracheal aspirate and CSF most common species isolated was Candida tropicalis. In blood cultures out of 19 maximum isolates were Candida tropicalis $7(36 \%)$.

Among the positive isolates Candida albicans was the most common species identified. Among the 102 Candida isolates, Candida albicans were $47(46 \%)$ in numbers followed by non albicans Candida were 55 (53.9\%)as shown in Table 3.

Out of 102 Candida isolates $52(51 \%)$ patients presented with fever on the other hand 50 (49\%) patients did not manifest fever.

Candida species were identified by Conventional methods those species were confirmed by growing them on HICHROME agar. All species were grown on Hichrome. Table 4 shows different Candida species on
Hichrome agar. HiChrome agar was used as differential medium for speciation of Candida isolates. HiChrome agar is an effective and fast screening agar for speciation of Candida. Both of the medium shows growth on both medium.

Table 5 shows antifungal sensitivity testing of Candida albicans. All strains of Candida albicans were susceptible to Amphotericin B. While out of 47 Candida albicans only $30(63.8 \%)$ shows sensitivity to Fluconazole and $25(71.4 \%)$ shows sensitivity to Itraconazole. Resistant to Candida albicans by Fluconazole $17(36 \%)$ and Itraconazole $22(47 \%)$ were noted. Itracanozole were found to be more resistant than fluconazole.

Table 6 shows showing antifungal sensitivity of Candida tropicalis. All strains of Candida tropicalis were susceptible to Amphotericin B. While out of 33 Candida tropicalis 28 $(84.8 \%)$ shows sensitivity to Fluconazole and $27(82 \%)$ shows sensitivity to Itraconazole. Resistant to Candida tropicalis by Fluconazole $5(15.1 \%)$ and Itraconazole 6 (18\%) were noted less in Candida tropicalis than Candida albicans.

Table.1 Shows Age groups of positive candida isolates

\begin{tabular}{|l|l|c|c|}
\hline Sr.no & Age groups & Total no. of patients & Percentage \\
\hline 1 & Baby & 13 & $12.7 \%$ \\
\hline 2 & $10-20 \mathrm{yrs}$ & 10 & $9.8 \%$ \\
\hline 3 & $20-30 \mathrm{yrs}$ & 10 & $9.8 \%$ \\
\hline 4 & $30-40 \mathrm{yrs}$ & 17 & $16.6 \%$ \\
\hline 5 & $40-50 \mathrm{yrs}$ & 13 & $12.7 \%$ \\
\hline 6 & 50 above & 39 & $38.2 \%$ \\
\hline
\end{tabular}


Table.2 Distribution of isolated Candida species in different samples

\begin{tabular}{|l|l|c|c|c|c|c|c|c|}
\hline Sr.no & $\begin{array}{l}\text { Name of Candida } \\
\text { species }\end{array}$ & Urine & Blood & Sputum & CSF & $\begin{array}{l}\text { Tracheal } \\
\text { aspirate }\end{array}$ & Pus & Total \\
\hline $\mathbf{1}$ & Candida.albicans & 29 & 6 & 7 & 2 & 1 & 2 & 47 \\
\hline $\mathbf{2}$ & Candida.tropicalis & 15 & 7 & 3 & 2 & 3 & 3 & 33 \\
\hline $\mathbf{3}$ & Candida.glabrata & 6 & 3 & 1 & 0 & 0 & 3 & 13 \\
\hline $\mathbf{4}$ & Candida. Krusei & 3 & 2 & 0 & 0 & 0 & 0 & 5 \\
\hline $\mathbf{5}$ & Candida.parapsilosis. & 2 & 1 & 0 & 0 & 0 & 0 & 3 \\
\hline $\mathbf{6}$ & Candida.dublinensis & 1 & 0 & 0 & 0 & 0 & 0 & 1 \\
\hline & Total & 56 & 19 & 11 & 4 & 4 & 8 & 102 \\
\hline
\end{tabular}

Table.3 The distribution of Candida albicans and non albicans Candida

\begin{tabular}{|l|l|c|c|}
\hline Sr.no & Candida isolates & No. of isolates & Percentage \\
\hline 1 & Candida albicans & 47 & $46 \%$ \\
\hline 2 & Non albicans Candida & 55 & $53.9 \%$ \\
\hline & Total & 102 & \\
\hline
\end{tabular}

Table.4 Comparison of conventional method of Candida species on SDA and Hichrome agar

\begin{tabular}{|l|l|c|c|}
\hline Sr.no & Candida species & Conventional method & Hichrome agar \\
\hline 1 & Candida.albicans & 47 & 47 \\
\hline 2 & Candida.tropicalis & 33 & 33 \\
\hline 3 & Candida.glabrata & 13 & 13 \\
\hline 4 & Candida.krusei & 5 & 5 \\
\hline 5 & Candida.parapsilosis. & 3 & 3 \\
\hline 6 & Candida.dublinenesis & 1 & -- \\
\hline
\end{tabular}

Table.5 Showing Antifungal sensitivity pattern of Candida albicans

\begin{tabular}{|l|l|c|c|}
\hline Sr.no & Antifungals & Sensitive & Resistant \\
\hline 1 & Fluconazole & $30(64 \%)$ & $17(36 \%)$ \\
\hline 3 & Amphotericin B & 47 & --- \\
\hline 4 & IT & $25(53 \%)$ & $22(47 \%)$ \\
\hline & Total & 47 & \\
\hline
\end{tabular}

Table.6 Showing antifungal sensitivity of Candida tropicalis

\begin{tabular}{|c|c|c|c|}
\hline Sr.no & Antifungals Agents & Sensitive & Resistant \\
\hline 1 & Fluconazole & $28 \quad(84.8 \%)$ & $5(15.1 \%)$ \\
\hline 2 & Amphotericin B & 33 & - \\
\hline 4 & Itraconazole & $27 \quad(82 \%)$ & $6(18 \%)$ \\
\hline & Total & 33 & \\
\hline
\end{tabular}


Table.7 Showing antifungal sensitivity of Candida glabrata

\begin{tabular}{|l|l|c|c|c|}
\hline Sr.no & Antifungals & Sensitive & \multicolumn{2}{|c|}{ Resistant } \\
\hline 1 & Fluconazole & $12(92.3 \%)$ & 1 & $(7.6 \%)$ \\
\hline 2 & Amphotericin B & 13 & & - \\
\hline 3 & Itraconazole & $10(77 \%)$ & 3 & $(23 \%)$ \\
\hline & Total & 13 & & \\
\hline
\end{tabular}

Table 7 showing antifungal sensitivity of Candida glabrata. All strains of Candida glabrata were susceptible to Amphotericin B. While out of 13 Candida glabrata 12 (92.3\%) shows sensitivity to Fluconazole and $10(77 \%)$ shows sensitivity to Itraconazole. Resistant to Candida glabrata by Fluconazole 1(7.6\%) and Itraconazole 3(23\%) were noted less in Candida glabrata than Candida albicans. and Candida tropicalis.

All species of Candida krusei, Candida parapsilosis and Candida dublinenesis were sensitive to Amhotericin B, Fluconazole and Itraconazole.

In the present study Candida albicans is the predominant species isolated from patients in urine and sputum samples similar to the study by Jayapriya et al (2012).

Along with Candida albicans the other Non albicans Candida species were also isolated. When taken into consideration both non albicans Candida was reported at a higher rate than Candida albicans. Among non-albicans Candida, Candida tropicalis was the most common species isolated in blood and pus in our study which is similar to study Ishita pandit et al., Increased use of antifungal drugs, use of antibiotics, long term use of catheters has contributes to emergence of nonalbicans Candida species. Deoruhkar et al (2013).

Candida isolates were seen in maximum in the age group above 50 years of age similar age group was also affected in study by Bhattacharjee et al., (2016). Advanced age also contributes to major risk factors associated with Candida.
Out of 102 isolates most of Candida isolates were from urine samples, followed by Blood, Sputum, Pus, Tracheal aspirate and CSF. Candida albicans (52\%) was isolated in maximum number in Urine and Sputum samples. In urine samples nonalbicans Candida were also isolated which were Candida tropicalis, Candida krusei and Candida glabrata. Most of the patients were catheterized. Insertion of in dewelling catheter for longer period might have been associated with Candidial infections.

In the present study, Conventional method and Hichrome differential agar were used for identification of Candida isolates. All species grown on SDA agar, by germ tube, on corn meal agar were accurately identified by Hichrome agar similar findings were shown by study Mangalkar et al., (2015). Among the newer test Hichrome agar is easy, effective and rapidly detecting medium identifying Candida species in $24-48$ hours.

Antifungal resistance to Itracanozole and Fluconazole were noted to Candida species in our study. More over Itraconazole showed more resistance than Fluconazole. Therefore Antifungal sensitivity testing is essential for administrating accurate antifungal drugs. By Morrell et al (2005)

In conclusion the candidial infection occurs in hospitalized patients. Non albicans Candida specially Candida tropicalis is also the causative agent followed by other species. Resistance to antifungal drugs among various Candida species is also noted. Indiscriminate use of azole group of drugs leads to resistance. Conventional method takes many days for 
Candida species identification. HiChrome agar can be used for rapid identification. Earlier detection of species and antifungal sensitivity testing can be used for earlier treatment and can save patients life.

\section{References}

Bhattacharjee, P. 2016. Epidemiology and antifungal susceptibility of Candida species in a tertiary care hospital, Kolkata, India. Curr Med Myco. 20-27.

Clinical Laboratory Standards Institute (CLSI), 2009. Method for antifungal disk diffusion susceptibility testing of yeasts. Approved guidelines. 2nd edn, CLSI document - M44 - A2, 29(17).

Deoruhkar S, Saini S. Nonalbicans candida species: Its isolation pattern, species distribution, virulence factors and antifungal susceptibility profile. Int $\mathrm{J}$ Med Sci Public Health 2013; 2(3): 5338.

Ishan Pandita, A.S. Wyawahare, M.V. Muley, Tazammul Hussain and Smita Kulkarni. 2019. Speciation and Antifungal Susceptibility pattern of Candida isolates from various clinical specimen at a tertiary care hospital Maharashtra. Int. J. Adv. Res. 7(10), 244-250.

Jayapriya Sukumaran, Jeya Meennakkshi Sundaram, Ranjith Raj Sivan. Changing trend in the clinical distribution of Candida species in a tertiary care hospital. 2012 Vol 1 /4 / page.no 222226.

Lewis R.E Overview of the changing epidemiology of Candidaemia. Curr. Med. Res. Opin. 2009; 25: 1732-1740. doi:10.1185/03007990902990817.

Mangalkar S M, Sagar K B, Gohel T and Sayare PC. Identification, speciation of Candida using chrome agar and its antifungal susceptibility testing in various clinical samples. International Journal of Recent Trends in Science And Technology, Volume 16, Issue 2, 2015 pp 440-445

Morrell M, Fraser VJ, Kollef MH. Delaying the empiric treatment of Candida bloodstream infection until positive blood culture results are obtained: A potential risk factor for hospital mortality. Antimicrob Agents Chemother 2005; 49:36405.

Roopa, C., S.Biradar. 2015. Isolation of Candida and its Speciation in Various Samples in a Tertiary Care Hospital in North Karnataka, India. Int.J.Curr.Microbiol.App.Sci. 4(9): 9961000.

Sanglard D. 2016. Emerging Threats in Antifungal-Resistant Fungal Pathogens. Front. Med. (Lausanne) 2016; 3: 11. doi: 10.3389/fmed..00011.

Sardi, J. C. O., L. Scorzoni, T. Bernardi, A. M. Fusco-Almeida, and M. J. S. Mendes Giannini, Candida species current epidemiology., pathogenicity, biofilm formation, natural antifungal products and new therapeutic options, Journal of Medical Microbiology, vol. 62, no. 1, pp. 10-24, 2013.

Shivprakash, S., Radhakrishnan, K., Karim, P. 2007. Candida spp. Other than Candida albicans: A major cause of fungaemia in a tertiary care centre. Indian $\mathbf{J}$ Med Microbiol. 25: 405-7.

\section{How to cite this article:}

Mangala S. Harbade, Jyoti A. Iravane, Shravani Kante and Nishigandha Pedwal. 2020. Identification of Candida Species and Antifungal Sensitivity Testing by Disc Diffusion Method. Int.J.Curr.Microbiol.App.Sci. 9(11): 2430-2436. doi: https://doi.org/10.20546/ijcmas.2020.911.292 